\title{
Religiöse Bildung in einer globalisierten Welt in postkolonialer Perspektive - Herausforderungen für die Islamische Religionspädagogik
}

\begin{abstract}
Einleitung
Globalisierung zeigt sich an unterschiedlichen Phänomenen und in unterschiedlichen Dimensionen. Eine Folge von Globalisierung ist Migration. Durch Migration werden Räume entgrenzt, was zu einer kulturellen und religiösen Pluralität sowie zur Zunahme von Heterogenität führt (vgl. Asbrand \& Scheunpflug, 2005, S. 268). „Die Vielfalt von Religionen und religiösen Orientierungen ist [...] eines der konstitutiven Elemente migrationsbedingt pluraler Gesellschaften " (Karakaşoğlu \& Klinkhammer, 2016, S. 298).

Aus einer religionspädagogischen Perspektive ist der Umgang mit unterschiedlichen religiösen und weltanschaulichen Vorstellungen, Haltungen und Einstellungen ein wesentliches Lern- und Bildungsziel. Die öffentliche Wahrnehmung des Islam spielt vor allem nach den islamistischen Anschlägen von 9/11 eine bedeutsame Rolle für den pädagogischen Diskurs um die Anderen, da diese Anschläge eine „Erschütterung einer als sicher geglaubten „westlichen Weltordnung" ausgelöst haben (ebd., S. 299). Auf die Aspekte der Differenzsetzung und Grenzziehungsdynamiken wird im folgenden Beitrag der Fokus gelegt. Dabei wird aus postkolonialer Perspektive das Phänomen des Otherings in den Blick genommen.

Die Islamische Religionspädagogik muss sich in kritischer Weise mit hegemonialen Ausprägungen von Diskursen in Bezug auf Religion in der Welt und Prozesse des Otherings auseinandersetzen, sie verstehen und bildungstheoretisch verarbeiten. Dabei kann die postkoloniale Theorie einen Zugang bieten, da sich diese Theorie mit der epistemischen Gewalt befasst, die die koloniale Herrschaft sicherte und moralisch legitimierte. „Untersucht wird deswegen im Kontext der postkolonialen Theorie eine Gewalt, die direkt mit der Wissensproduktion und auch -vermittlung im Zusammenhang steht. Insoweit werden pädagogische Fragestellungen unmittelbar davon berührt" (Castro Valera, 2016, S. 153).

Es stellt sich die Frage, welche Auswirkungen Grenzziehungsdynamiken der Akteur/-innen auf Seiten der Mehrheitsgesellschaft und auf Seiten der ausgegrenzten Gruppe (hier die muslimische Minderheit) für die Bildungslandschaft haben. Religionspädagog/-innen sind herausgefordert, bei der Gestal-
\end{abstract}

tung von Lernlandschaften die Spannungsverhältnisse aufzugreifen und Impulse anzubieten, die weder zu Selbst- noch zu Fremdausgrenzungen führen. Das beinhaltet aber auch, problematische Überzeugungshaltungen (z.B. rassistische, fundamentalistische, extremistische Werthaltungen) bei Bildungspartizipierenden entgegenzuwirken und zwar durch eine diskriminierungskritische und differenzsensible religionspädagogische Arbeit, die nicht einseitig, sondern symmetrisch ist.

\section{Globalisierung und transnationale Migration}

Nach Naika Foroutan ist transnationale Migration ein selbstverständliches Zeichen der globalisierten Welt (Foroutan 2010, S. 9). Migration löst „Prozesse der Transformation natioethnokultureller Selbstverständnisse von Individuen und Gesellschaft[en] aus" bzw. verstärkt diese (Karakaşoğlu \& Klinkhammer, 2016, S. 294).

Mindestens $20 \%$ der in Deutschland lebenden Menschen, ihre Eltern oder Großeltern haben Migrationserfahrung. Ein großer Teil der Migrant/-innen hat eine muslimische Religionszugehörigkeit, aber auch Angehörige des orthodoxen Christentums machen einen nicht unerheblichen Anteil aus. Bei einem Drittel der Menschen mit Migrationshintergrund ist Migration sogar keine selbsterlebte Erfahrung mehr. Aber durch Merkmale wie Aussehen, Kleidung oder Namen bleibt bei ihnen Migration als ein „Element der biografischen Kernnarration" bestehen (Foroutan, 2010, S. 10).

Der aktuelle Policy Brief „Wo kommen Sie eigentlich ursprünglich her?" des Forschungsbereichs beim Sachverständigenrat deutscher Stiftungen für Integration und Migration (SVR) macht deutlich, dass unabhängig von der Herkunft sich Menschen mit sichtbarem Migrationshintergrund häufiger diskriminiert fühlen als Zugewanderte, deren Erscheinungsbild sich nicht durch natürliche Merkmale wie Hautfarbe oder kulturelle Merkmale wie Kopftuch von der Mehrheitsbevölkerung unterscheidet (vgl. SVR-Forschungsbericht 2018). Die Untersuchung zeigt, dass Menschen mit sichtbarem Migrationshintergrund zu $48 \%$ von erlebter Benachteiligung berichten. Die 
Prozentzahl steigt, wenn sie zusätzlich mit Akzent Deutsch sprechen, sie sinkt auf $17 \%$, wenn sich Zugewanderte äußerlich nicht von der Mehrheitsbevölkerung unterscheiden. Die Religionszugehörigkeit spielt ebenfalls eine Rolle. So berichten Zugewanderte muslimischen Glaubens deutlich häufiger von Diskriminierung (55\%) als Zugewanderte mit christlicher (29 \%) oder ohne Religionszugehörigkeit (32 \%) (vgl. ebd.). Das kann darauf zurückgeführt werden, dass das Verhältnis von Religionen in der Alltagswelt vorstrukturiert ist: „Es gibt in der öffentlichen Wahrnehmung und Darstellung ,gute ${ }^{\text {‘ }}$ und ,schlechte` Religionen, d.h. Grenzziehungen sowie Zuordnungsschemata, die unabhängig von der Bedeutung von Religion und Religiosität für die Einzelnen greifen. Der Islam steht in dieser Ordnungspolitik derzeit, anders als der Buddhismus oder Hinduismus, ganz auf der negativen Seite“ (Karakaşoğlu \& Klinkhammer, 2016, S. 296).

\section{Postkoloniale Theorie und ihre Perspektiven für Bildung}

Die heutige Situation in Europa wird durch das Erbe des Kolonialismus stark beeinflusst: „Vom Klimawandel bis zur angeblichen ,Flüchtlingskrise‘, von den gesellschaftlichen Kontroversen um die Rolle von Religion bis hin zu Fragen von Minderheitenrechten und Staatsbürgerschaft: überall hat die Jahrhundertelange koloniale Herrschaft Spuren hinterlassen, die in der gegenwärtigen geopolitischen Situation vielleicht sichtbarer denn je sind“" (Castro Valera \& Dhawan, 2020, S. 7f.). So plädieren María do Mar Castro Valera und Nikita Dhawan dafür, postkoloniale Perspektiven in wissenschaftlichen Arbeiten nicht mehr zu vernachlässigen, um die Interdependenzen und Verflechtungen zwischen „Vergangenheiten und Zukünften“ zu verstehen und die Auswirkungen der rassistischen und kolonialistischen Vergangenheit auf unsere Gegenwart ernst zu nehmen (ebd., S. 8). Kolonialismus und der damit zusammenhängende Imperialismus sind nicht als europäisches Phänomen zu denken, sondern als ein europäisches und außereuropäisches Gesamtphänomen, wobei die historischen Prozesse nicht als lineare Entwicklungen betrachtet werden, sondern als komplexe Prozesse und Praktiken mit Brüchen und Widersprüchen (vgl. ebd., S. 24).

Postkoloniale Theorie analysiert „die hegemoniale Wissensproduktion, die epistemische Gewalt und [...] die normalisierte Produktion von Bildungsferne" (Castro Valera, 2016, S. 152). Für die (religions)pädagogische Reflexion im Zeitalter der Globalisierung ist das Zusammenspiel von Bildung und Macht wesentlich, denn in dieser Perspektive ist Wissen immer gebunden an bestimmte Macht- und Herrschaftsstrukturen. Von den gesellschaftlichen Macht- und Herrschaftsstrukturen hängt es ab, wer in der hegemonialen Position ist, Menschen entlang bestimmter Merkmale zu markieren und sie auf diese Merkmale stereotypisierend und essentialisierend zu reduzieren. Die Bilder und Stereotype der hegemonialen Position finden Eingang in das "gesellschaftlich ,gültige“ Wissen" (Foroutan \& İkiz, 2016, S. 143). Diese stereotypen Bilder sind nach Naika Foroutan und Dilek İkiz soziale Repräsentationen, in denen Bedeutungen in einem sozialen Prozess diskursiv produziert werden. „Die Wirkmächtigkeit von Diskursen zeigt sich darin, dass sie über Wissen machtvoll, Wahrheiten ' produ- zieren und transportieren, und somit die Wahrnehmung und Interpretation von sozialer, Wirklichkeit' beeinflussen“" (ebd.). Die Dominanzverhältnisse in einer Gesellschaft haben hegemoniale Wissensproduktion und epistemische Gewalt zur Folge, die vor allem im Bereich der Bildung destruierende Folgen haben können.

Edward Said hat in seinem Werk Orientalism (vgl. Said, 1978) eindrücklich dargelegt, „wie die Herstellung und Bereitstellung eines spezifischen Wissens der kolonialen Beherrschung dienlich war und gleichzeitig derselben ermöglichte, ein Wissen über den Orient zu etablieren, das den Orient als Antagonist des Okzidents diskursiv hervorbrachte. In Konsequenz stand fortan das Bild eines ,barbarischen und unberechenbaren Orients einem des ,zivilisierten und kalkulierbaren Okzidents' gegenüber" (Castro Valera, 2016, S. 153-154). Orientalismus ist nach Said ein hegemonialer europäischer Diskurs, der den Einheimischen neu definiert und damit imperiale Macht demonstriert. Said verwendete das Konzept des „Othering“ (Zum-Anderen-machen), mit dem er zeigt, wie das europäische „wir“ als überlegen und souverän und das orientalische „Andere“ als different und unterlegen konstruiert und festgesetzt werden (vgl. ebd., S. 154). Damit werden die anderen zu einem Objekt gemacht, das untersucht werden kann. In der Beschreibung des Untersuchungsobjekts sind nach Said die hegemonialen Vorstellungen Europas eingeflossen, die die Vorstellungen von Ländern wie Ägypten, Indien oder auch der Türkei bestimmen (vgl. ebd., S. 155). Das koloniale Wissen fand aber auch Eingang in das Selbstverständnis der Kolonisierten.

Hegemoniales Wissen, das durch eine machtvolle Bezeichnungs- und Abgrenzungspraxis gekennzeichnet ist und im gesellschaftlichen Diskurs konstruiert wird, muss nach Gayatri Chakravorty Spivak (vgl. Spivak, 1993) hinterfragt werden, um die Machteffekte darin zu analysieren, indem verdeutlicht wird, wie Wahrheiten und Weltsichten konstruiert wurden. Wissensproduktion ist also kein harmloses Unterfangen, sie ist auch nicht unhinterfragt zu übernehmen, sondern sie muss immer wieder einer Überprüfung unterzogen werden, denn Weltsichten sind immer verschiedene Lesarten und keine unumstößlichen Wahrheiten (vgl. Castro Valera, 2016, S. 161).

\section{Religiöses Othering}

Nicht zuletzt ist die Wahrnehmung der Muslim/-innen als „Andere“ stark durch orientalistische Zuschreibungen geprägt, die nach Said zu einer Teilung zwischen Ost und West geführt hat, die imaginativ war und dem Ziel der Beherrschung diente. Aufgrund der Fokussierung auf Religion wird der Orient in den letzten Jahren mit dem Islam gleichgesetzt, so Lingen-Ali und Mecheril. Dieser Prozess hat zur Folge, dass ein kollektives „Wir“ und ein kollektives „die Anderen“ anhand der Kategorie Religion konstruiert werden: „Subjekte werden so einem bestimmten religiösen Kollektiv (dem Islam) zugeordnet, sie werden als homogene Gruppe konstruiert, die als grundsätzlich anders als die eigene Gruppe wahrgenommen wird. Der Islam wird zur anderen, zur fremden Religion" (Lingen-Ali \& Mecheril, 2016, S. 19). Jugendliche werden mit Hilfe der ,sozialen Deutungs- und Identifikationspraxis Religion“ als Muslim/-innen hergestellt bzw. „muslimisiert“ oder muslimischer gemacht als sie sind. Das kann auch dazu führen, dass sie sich selbst „mus- 
limisieren“ (ebd., S. 20). Die „Muslimisierung“ muslimischer Migrantinnen und Migranten hat religiös und ethnisch aufgeladene Zuschreibungen zur Folge und fungiert als symbolische Differenzlinie zwischen „Wir“ und „Ihr“, auch im schulischen Raum (vgl. Lingen-Ali, 2013).

Die Epistemologie, die hinter dieser und anderen Fragen steckt, ist nach Lingen-Ali und Mecheril die Annahme, dass es hier eine essenzielle Differenz zum Anderen gibt (Islam \& Muslim/-innen), die wie auch immer bearbeitet werden muss (vgl. Lingen-Ali \& Mecheril, 2016, S. 20). Diese essenzielle Differenz zum Anderen wird auch über einen Gegensatz zum christlichen Glauben hergestellt, wie Rainer Möller zeigt: „In gesellschaftlichen Diskursen wird zunehmend populärwissenschaftliches Wissen über ,den' Islam als, fremder' Religion produziert und in einen fundamentalen Gegensatz zu säkularen Weltanschauungen und zum christlichen Glauben als der, eigenen ' Religion gestellt" (Möller, 2017, S. 48). Freilich gibt es auch differenzierte Diskurse, die weder den Islam verdinglichen noch Muslim/innen über einen Kamm scheren (vgl. z. B. Bauer, 2011, 2018; Griffel, 2018). Dennoch ist dieser populärwissenschaftliche Diskurs einer der dominantesten in Deutschland, der auch in institutionelle Kontexte und pädagogische Praxen eingeht, wie Forschungen zeigen (vgl. z.B. Kul, 2013; Lingen-Ali, 2012; Shooman, 2014; Rose, 2010; Nguyen, 2013).

Muslim/-innen sind in dieser Lesart eine homogene Gruppe, denen bestimme Merkmale zugewiesen werden. Der Islam ist ihr wesentliches Differenzierungsmerkmal, wodurch gesellschaftliche und soziale Verhältnisse begründet werden (vgl. Lingen-Ali/Mecheril 2016, S. 22). Nun finden sich Phänomene wie häusliche Gewalt, Homophobie, Unterdrückung von Frauen usw. natürlich auch bei Menschen mit muslimischer Religionszugehörigkeit. Und natürlich stehen solche Praktiken auch mit religiösen Einstellungen und Haltungen in Verbindung. Die verheerende Wirkung der Vorstellung des Islams als einer essenziell anderen Religion besteht darin, solche Phänomene auf die Kategorie Religion zu reduzieren, mit dem Muslimsein an sich gleichzusetzen und von einer in dieser angenommenen anderen Essenz verankerten Unveränderlichkeit dieser Konstruktion auszugehen. Durch eine Reduktion von Menschen mit muslimischem Glauben auf die Kategorie andere Religion, die ihrem Wesen vermeintlich inhärent ist, wird eine symbolische Grenze geschaffen, die Exklusion und Dominanz legitimiert, ganz ähnlich einer hegemonialen Zuordnungspraxis (ebd.). Innerislamische Diversität, die durch zahlreiche Studien belegt wird, wird im öffentlichen Diskurs ignoriert (vgl. z.B. Boos-Nünning/Karakaşoğlu 2006; Nökel 2007; Gerlach 2006; Tressat 2011). Andere Kategorien, wie Klasse, Geschlecht, Sexualität, Kultur, Ethnie oder ökonomische Verhältnisse werden bei der Konstruktion der „anderen Religion“ erst gar nicht in Betracht gezogen, weil ihre Irrelevanz angesichts der epistemischen Wucht des Konstrukts selbstevident erscheint.

\section{Herausforderungen für die Islamische Religionspädagogik}

Für die religionspädagogische Arbeit stellt sich die Frage, welche Auswirkungen Erfahrungen des Othering auf muslimische Kinder und Jugendliche haben und wie eine Islamische Religionspä- dagogik Lernlandschaften gestalten kann, um den Kindern und Jugendlichen Möglichkeiten zu schaffen, mit Grenzziehungsdynamiken und Stereotypenbildungen reflektiert und konstruktiv umzugehen.

Die Konstruktion von Islam und Westen als vermeintlich inkompatible Gegensätze, von denen einer höherwertiger, entwickelter, emanzipierter, zivilisierter, vernünftiger und moderner ist als der andere, kann ein dichotomes Selbst- und Fremdbild bei muslimischen Kindern und Jugendlichen erzeugen (vgl. Attia, 2013). Das kann dazu führen, dass sich diese muslimischen Kinder und Jugendlichen dementsprechend als eine Art kollektive muslimische Persönlichkeit verstehen, die die Konstruktion einer Fiktion von muslimischer Identität und einer muslimischen Kultur zur Folge hat. Die Reduzierung auf eine sog. muslimische Identität kann zu Widerstand und Rebellion führen und in Prozessen der Selbst- und Fremdausgrenzung enden.

Erfahrungen des Othering können dazu führen, dass der Islam auch als Differenzkategorie für muslimische Selbstabgrenzungen genutzt wird. Solche Selbstabgrenzungen haben zur Folge, dass sich religiöse „Wir-Gruppen“ („ingroup“) und „Sie-Gruppen“ („outgroup“) herausbilden. Die Gruppengrenzen werden durch „cultural stuff“ (Barth, 1969, S. 15) geschlossen, wie „Sprache, Ritual, Verwandtschaft, Lebensführung“" (Dahinden, Duemmler \& Moret, 2010, S. 2).

Eine Grenzziehung über die Kategorie Religion führt in der Tendenz dazu, die eigene Religion als höherwertiger anzusehen als die der Anderen. Das Individuum, das zu dieser „,ingroup " gehört, wertet sich auf. Das kann dazu frühen, dass Jugendliche empfänglich werden für die Rekrutierung durch religiös extremistische oder fundamentalistische Netzwerke.

Muslimische Religionslehrkräfte sind dazu aufgefordert, eine Lehrpraxis zu entwickeln, die sensibel ist für hegemoniale Wissensproduktion und epistemische Gewalt. Dazu gehört, sich mit religiösen Grenzziehungsdynamiken und Stereotypenbildungen auseinanderzusetzen, um zu analysieren, wie auf subjektiver, struktureller, institutioneller und diskursiver Ebene Diskriminierung auf der Grundlage von religiösem Othering auftreten kann. Zudem gehört es zur professionellen Selbstreflexion, sich der eigenen Rolle als machtvolle/-r Akteur/-in bewusst zu werden und das eigene Verhältnis zur Religion zu reflektieren (vgl. Karakaşoğlu \& Klinkhammer, 2016, S. 307).

Eine differenzsensible und diskriminierungskritische Lehrpraxis fragt danach, wie Ungleichheiten minimiert werden können. Allerdings darf dabei nicht außer Acht gelassen werden, dass bei einer differenzsensiblen Vorgehensweise Differenzen auch ungewollt reproduziert werden können: „Bildungsprozesse in einem von Differenzen präformierten Kontext (re-)produzieren Ungleichheiten, wenn diese Differenzen nicht erkannt und anerkannt werden. Auf der anderen Seite reproduziert die Anerkennung von Unterschieden in einer doppelten Weise Machtverhältnisse: Die Anerkennung der anderen [...] anerkennt sie als andere, die sie nur in einer hierarchischen Ordnung [...] werden konnten, wodurch paradoxerweise diese hegemoniale Ordnung bekräftigt und bestätigt wird“ (Mecheril \& Plößer, 2009, S. 20) Dieses Dilemma kann nicht aufgelöst werden, daher ist eine „kritisch-reflexive Thematisierung von Differenz" notwendig (ebd., S. 196). 
Unterrichtspraktisch wird empfohlen, Lernlandschaften zu konzipieren, mittels derer die Schüler/-innen die Möglichkeit erhalten, zunächst einmal Grenzziehungsdynamiken und Stereotypenbildungen wahrzunehmen. In einem zweiten Schritt ist es bedeutsam, zu hinterfragen, welche Auswirkungen diese Dynamiken des Otherings auf das eigene Selbstbild haben, und den sich daraus entwickelnden eigenen Konstruktionen des Anderen in ihrer gegenseitigen Abhängigkeit auf den Grund zu gehen. Der Unterricht soll darauf abzielen, auf Seiten der Schüler/-innen eine Fragehaltung hervorzubringen, die Fremd- und Selbstreflexionsprozesse auslöst.

Zudem sollen die Lernlandschaften den Schüler/-innen die Möglichkeit bieten, Ursachen und Konsequenzen von hegemonialer Wissensproduktion und epistemischer Gewalt zu analysieren, d. h., der Reproduktion von machtvollen Unterscheidungspraxen auf die Spur zu kommen und die Ordnungen, die sie hervorbringen, zu problematisieren.

Dabei ist es wesentlich, auch die wechselseitigen Prozesse des Otherings und die Konsequenzen, die daraus resultieren, bewusst zu machen und Strategien mit den Schüler/-innen zu entwickeln, wie sie jenseits von binären Selbst- und Fremdausgrenzungen auf Prozesse des Otherings reagieren können. Es ist bei aller Berechtigung nicht ausreichend, Kritik an hegemonialen Zuschreibungen zu üben, sondern es ist darüber hinaus erforderlich, einerseits selbstkritisch die eigenen Praktiken zu hinterfragen und andererseits den Versuch zu unternehmen, die binäre Logik zu durchschauen und zu aufzubrechen, die zu dieser epistemischen Gewalt geführt hat.

Das kann dadurch erreicht werden, dass ein anderes Vokabular jenseits der Dichotomien konstruiert wird, beispielsweise, indem man bewusst nicht mehr von Grenzen spricht, sondern z.B. von Schnittstellen oder indem man auf dichotome Terminologien verzichtet und für sie diskursorientierte, offenere Ersatzbegriffe sucht (vgl. Engelbrecht, 2009). Dichotomes Denken und Sprechen führt unweigerlich zu Übervereinfachungen und Ideologisierungen. Eine differenzsensible und diskriminierungskritische Sprache kann hier helfen, machtvoll erzeugte Differenzen zu überwinden und den Schüler/-innen neue Horizonte des Sprechens und Denkens zu eröffnen.

\section{Literatur}

Asbrand, B. \& Scheunpflug, A. (2005). Zum Verhältnis zwischen interreligiösem, interkulturellem, ökumenischem und globalem Lernen. In P. Schreiner, U. Sieg \& V. Elsenbast (Hrsg.), Handbuch interreligiöses Lernen (S. 268-282). Gütersloh: Gütersloher Verlagshaus.

Attia, I. (2013). Privilegien sichern, nationale Identität revitalisieren. Gesellschaftsund handlungstheoretische Dimensionen der Theorie des antimuslimischen Rassismus im Unterschied zu Modellen von Islamophobie und Islamfeindlichkeit. Journal für Psychologie 21, 1-31.

Barth, F. (1969). Ethnic groups and boundaries: the social organization of culture difference. Bergen: Univ. Forl.

Bauer, T. (2011). Die Kultur der Ambiguität. Eine andere Geschichte des Islams. Berlin: Verlag der Weltreligionen.

Bauer, T. (2018). Warum es kein islamisches Mittelalter gab: Das Erbe der Antike und der Orient. München: C.H.Beck. https://doi.org/10.17104/9783406727313

Boos-Nünning, U. \& Karakaşoğlu, Y. (2006). Viele Welten leben: Zur Lebenssituation von Mädchen und jungen Frauen mit Migrationshintergrund (2. Auflage). Münster: Waxmann.

Castro Varela, M. (2016). Postkolonialität. In P. Mecheril (Hrsg.), Handbuch Migrationspädagogik (S. 152-166). Weinheim u. Basel: Beltz.
Castro Varela, M. \& Dhawan, N. (2020). Postkoloniale Theorie (3., aktualisierte Auflage). Bielefeld: Transkript.

Dahinden, J., Duemmler, K. \& Moret, J. (2010). Religion und Ethnizität: Welche Praktiken, Identitäten und Grenzziehungen. In SOFIS - Sozialwissenschaftliche Forschungsinformationen. http://p3.snf.ch/project-115756

Engelbrecht, M.(2009). Von Konfessionen und Religionen zu Diskursen und Netzwerken. Innen- und Außensichten religiöser Welten. In J. Först \& H. G. Schöttler (Hrsg.), Quo vadis, theologia? (S. 71-91). Berlin: LIT.

Foroutan, N. (2010). Neue Deutsche, Postmigranten und Bindungs-Identitäten. Wer gehört zum neuen Deutschland? In Bundeszentrale für politische Bildung: Aus Politik und Zeitgeschichte. Anerkennung, Teilhabe, Integration, 9-15.

Foroutan, N. \& İkiz, D. (2016). Migrationsgesellschaft. In P. Mecheril (Hrsg.), Handbuch Migrationspädagogik (S. 138-152). Weinheim u. Basel: Beltz.

Gerlach, J. (2006). Zwischen Pop und Dschihad. Muslimische Jugendliche in Deutschland. Berlin: Ch. Links.

Griffel, F. (2018). Den Islam denken. Versuch, eine Religion zu verstehen. Ditzingen: Reclam.

Karakaşoğlu, Y. \& Klinkhammer, G. (2016). Religionsverhältnisse. In P. Mecheril (Hrsg.), Handbuch Migrationspädagogik (S. 294-310). Weinheim u. Basel: Beltz.

Kul, A. (2013): „Jetzt kommen die Ayşes auch ins Lehrerzimmer und bringen den Islam mit". Subjektiv bedeutsame Erfahrungen von Referendarinnen und Referendaren im Rassismuskontext. In K. Bräu et al. (Hrsg.), Lehrerinnen und Lehrer mit Migrationshintergrund. Zur Relevanz eines Merkmals in Theorie, Empirie und Praxis (S. 157-175). Münster: Waxmann.

Lingen-Ali, U. (2012). ,Islam`als Zuordnungs- und Differenzkategorie. Antimuslimische Ressentiments im Bereich von Bildung und Sozialer Arbeit. In Sozial extra, 12(9/10), 24-27. https://doi.org/10.1007/s12054-012-1008-4

Lingen-Ali, U. (2013). Die ,andere Religion: Muslimisierung und Selbstpositionierung - Wie der Islam (nicht nur) im Religionsunterricht als Differenzlinie fungiert. In R. Leiprecht \& A. Steinbach (Hrsg.), Schule in der Migrationsgesellschaft. Ein Handbuch (S. 324-341). Schwalbach/Taunus: Wochenschau.

Lingen-Ali, U. \& Mecheril, P. (2016). Religion als soziale Deutungspraxis. In $\ddot{O}_{s-}$ terreichisches Religionspädagogisches Forum (S. 17-24). Zugriff am 16.09.2020 https://www.fachportal-paedagogik.de/literatur/vollanzeige.html?FId=1127803\#vollanzeige.

Mecheril, P. \& Plößer, M. (2009). Differenz. In S. Andresen et al. (Hrsg.), Handwörterbuch Erziehungswissenschaft (S. 194-208). Weinheim u. Basel: Beltz.

Möller, R. (2017). Interreligiöse Bildung aus migrationsgesellschaftlicher Perspektive. In R. Möller, C. P. Sajak \& M. Khorchide (Hrsg.), Kooperation im Religionsunterricht. Chancen und Grenzen interreligiösen Lernens: Beiträge aus evangelischer, katholischer und islamischer Perspektive (S. 43-59). Münster: Comenius-Institut.

Nguyen, T. Q. (2013). „Es gibt halt sowas wie einen Marionettentäter“. Schulischinstitutionelle Rassismuserfahrungen, kindliche Vulnerabilität und Mikroaggressionen. In: ZEP, 36(2), 20-24.

Nökel, S. (2007). „Neo-Muslimas“ - Alltags- und Geschlechterpolitiken junger muslimischer Frauen zwischen Religion, Tradition und Moderne. In H. J. v. Wensierski \& C. Lübcke (Hrsg.), Junge Muslime in Deutschland: Lebenslagen, Aufwachsprozesse und Jugendkulturen (S. 135-154). Opladen: Barbara Budrich. https://doi. org/ $10.2307 /$ j.ctvddzp4d.10

Rose, N. (2010). Differenz-Bildung. Zur Inszenierung von Migrationsanderen im schulischen Kontext. In A. Broden \& P. Mecheril (Hrsg.), Rassismus bildet (S. 209233). Bielefeld: Transcript.

Said, E. W. (1978). Orientalism. New York: Pantheon.

Shooman, Y. (2014). „... weil ihre Kultur so ist. “ Narrtive des antimuslimischen Rassismus. Bielefeld: Transcript. https://doi.org/10.14361/transcript.9783839428665 Spivak, G. C. (1993). Outside in the Teaching Machine. New York: Routledge.

SVR-Forschungsbericht (2018). „Wo kommen Sie eigentlich ursprünglich her?" Diskriminierungserfahrungen und phänotypische Differenz in Deutschland. Berlin: Sachverständigenrat deutscher Stiftungen für Integration und Migration. (= Policy Brief des SVR-Forschungsbereichs).

Tressat, M. (2011). Muslimische Adoleszenz? Frankfurt a. M. u. a.: Lang.

\section{Dr. Fahimah Ulfat}

ist Professorin für Islamische Religionspädagogik am Zentrum für Islamische Theologie der Eberhard-Karls-Universität Tübingen. Ihre Forschungsschwerpunkte sind empirische Unterrichtsforschung, Professionsforschung und Forschung zu Jugend und Religion. 